\title{
New progress in the study of germline susceptibility genes of myeloid neoplasms (Review)
}

\author{
LEI BI ${ }^{1}$, TIANYUAN MA ${ }^{2 *}, \mathrm{XU} \mathrm{LI}^{3^{*}}, \mathrm{LAI} \mathrm{WEI}^{3 *}, \mathrm{ZINUO} \mathrm{LIU}^{3 *}$, \\ BINGYUE FENG ${ }^{3}$, BAOXIA DONG ${ }^{1}$ and XIEQUN CHEN ${ }^{1,4}$ \\ ${ }^{1}$ Department of Hematology, Xijing Hospital, Fourth Military Medical University; \\ ${ }^{2}$ Department of Stomatology, Fourth Military Medical University; ${ }^{3}$ College of Basic Medicine, \\ Fourth Military Medical University, Xi'an, Shaanxi 710032; ${ }^{4}$ Hematology and Oncology Center, \\ Affiliated Hospital of Northwest University and Xian No. 3 Hospital, Xi'an, Shaanxi 710082, P.R. China
}

Received October 10, 2020; Accepted January 21, 2021

DOI: 10.3892/ol.2021.12578

\begin{abstract}
In 2016, the World Health Organization incorporated 'myeloid neoplasms with germline predisposition' into its classification of tumors of hematopoietic and lymphoid tissues, revealing the important role of germline mutations in certain myeloid neoplasms, particularly myelodysplastic syndrome and acute myeloid leukemia. The awareness of germline susceptibility has increased, and some patients with myeloid neoplasms present with a preexisting disorder or organ dysfunction. In such cases, mutations in genes including CCAAT enhancer binding protein $\alpha$ (CEBPA), DEAD (Asp-Glu-Ala-Asp) box polypeptide 41 (DDX41), RUNX family transcription factor 1 (RUNX1), GATA binding protein 2 (GATA2), Janus kinase 2 (JAK2) and ETS variant transcription factor 6 (ETV6) have been recognized. Moreover, with the application of advanced technologies and reports of more cases, additional germline mutations associated with myeloid neoplasms have been identified and provide insights into the formation, prognosis and therapy of myeloid neoplasms. The present review discusses the well-known CEBPA, DDX41, RUNX1, GATA2, JAK2 and ETV6 germline mutations, and other mutations including those of lymphocyte adapter protein/SH2B adapter protein 3 and duplications of autophagy related 2B, GSK3B interacting protein and RB binding protein 6 , ubiquitin ligase, that remain to be confirmed or explored. Recommendations for the
\end{abstract}

Correspondence to: Professor Baoxia Dong or Professor Xiequn Chen, Department of Hematology, Xijing Hospital, Fourth Military Medical University, 127 Chang'le West Road, Xi'an, Shaanxi 710032, P.R. China

E-mail: freshupdbx@hotmail.com

E-mail: xiequnchen@fmmu.edu.cn

*Contributed equally

Key words: myeloid neoplasms, myeloid dysplastic syndrome, acute myeloid leukemia, myoproliferative neoplasms, germline mutations management of diseases associated with germline mutations are also provided.

\author{
Contents \\ 1. Introduction \\ 2. Germline mutation of myeloid neoplasms \\ 3. Clinical significance \\ 4. Summary and prospects
}

\section{Introduction}

Myeloid neoplasms are a group of heterogeneous neoplasms formed by the clonal proliferation of hematopoietic stem cells (HSCs) with multidirectional differentiation potential in the bone marrow, and they include acute myeloid leukemia (AML), myelodysplastic syndrome (MDS) and myeloproliferative neoplasms (MPNs). The pathogenesis of myeloid neoplasms is complicated, and germline mutations play a critical role in this process. Patients with these mutations have been observed to have a predisposition to MDS/AML and other myeloid neoplasms. This concept was added to the 2016 revision to the World Health Organization (WHO) classification of myeloid neoplasms and acute leukemia as a new category, indicating the importance of germline mutations in these hematopoietic malignancies (1). This revision is profound, as patients with myeloid neoplasms caused by germline mutations may have different clinical manifestations, responses to clinical management and prognoses from those with de novo MDS and AML (2). Patients with MDS/MPNs who have germline mutations also have a higher risk of developing AML, which is also likely to have more malignant clinical features and poor outcomes. Therefore, it is essential to identify the germline mutations in myeloid neoplasms, understand their mechanisms and take early therapeutic measures with long-term follow-ups. Myeloid neoplasms with germline mutations are sporadic, but with the application of whole-genome and targeted sequencing and more familial hematopoietic disorders being reported, a greater number of germline mutations have been identified 
and more clearly defined. The present review summarizes basic information about germline mutations, and the features of associated clinical syndromes or diseases in Tables I and II.

\section{Germline mutation of myeloid neoplasms}

In the 2016 revision of the WHO classification guidelines for myeloid neoplasms and acute leukemia (1), the molecular recognition of germline predisposition to hematopoietic neoplasms was formalized. For example, the primary genetic susceptibility factors used to identify bone marrow malignancies, namely RUNX family transcription factor 1 (RUNX1), CCAAT enhancer binding protein $\alpha$ (CEBPA) and GATA binding protein 2 (GATA2), are hematopoietic transcription factors, which are often associated with the onset of malignancies in young individuals. While idiopathic MDS has a typical onset age of $>60$ years, it is common for patients with GATA2 deficiency to develop MDS at a younger age, with a median age at onset of 29 years; in these patients, the prevalence of MDS during their lifetime is estimated to be $90 \%$, and GATA2-associated MDS is a high-risk pre-leukemic disease that may rapidly develop into AML (3). The recent identification of DEAD (Asp-Glu-Ala-Asp) box polypeptide 41 (DDX41) germline mutations in familial myeloid malignancies has also led to a shift in the view of susceptibility to hematologic malignancies, indicating an association of germline mutations with age at onset and potential function. Therefore, further research into the myeloid neoplasms associated with germline mutations is recommended.

CEBPA. The CEBPA gene is located on chromosome $19 \mathrm{q} 13.1$, and encodes a transcription factor that is essential for granulocytic differentiation and cellular growth arrest $(4,5)$. CEBPA consists of three domains, which comprise two $\mathrm{N}$-terminal transactivating domains, and a basic region with leucine-zipper (bZIP) for specific DNA sequence binding and dimerization, respectively, at the C-terminal end (6). The bZIP domain of the transcription factor is able to recognize the CCAAT motif in the promoters of target genes (4). Alternative in-frame non-AUG (GUG) and AUG start codons result in protein isoforms with different lengths, namely $\mathrm{p} 42$ and $\mathrm{p} 30$. CEBPA-p42, as the full-length isoform, has been widely studied, and its function has been shown to be associated with the proliferation and differentiation of myeloid progenitors (7). A study by Zhang et al (8) found that the loss of CEBPA-p42 in mice disrupted the normal development of terminally differentiated granulocytes and macrophages, and increased the self-renewal of HSCs. The truncated protein CEBPA-p30 is a negative factor responsible for blocking differentiation in AML, and mutations in CEBPA are critical in the disruption of myeloid differentiation in AMLs (9).

Smith et al (6) reported the first case of CEBPA mutation in 2004. The case was a member of a family in which three members were affected by AML. The researchers identified a 212delC mutation in CEBPA by analyzing DNA extracted from peripheral blood samples. Two siblings possessed a normal karyotype and were diagnosed with a rare disease, namely M2 with eosinophilia, and their father was diagnosed with M1. The researchers screened for the 212 delC mutation in five healthy family members and found the wild-type sequence, indicating that the germline CEBPA mutation truly contributed to the development of AML. The characteristics of these three familial cases were consistent with French-American-British subtype M1 or M2 classification.

Sellick et al (10) reported further cases with a germline mutation in CEPBA that caused AML, supporting the previous conclusion. The authors screened five members of a family, three of whom were diagnosed with AML. The affected patients were found to harbor an out-of-frame germline 217ins $\mathrm{C}$ mutation in the $\mathrm{N}$-terminal area of CEBPA, leading to reduced expression of the $42-\mathrm{kDa}$ isoform and enhanced production of the $30-\mathrm{kDa}$ isoform. Corresponding results were observed in two subsequent studies $(11,12)$. It is worthy of note that the locations and types of mutations found by Sellick et al (10) are almost identical to the mutations in the aforementioned family with AML, indicating that these mutation of CEBPA may serve to initiate the pathogenesis of AML as a dependent factor.

In subsequent years, other germline CEBPA mutations have been reported, including 350-351insCTAC, 291delC, 465-466insT and 217-218insC (11-13). All of these mutations are located in the $\mathrm{N}$-terminal region. Yan et al (14) also reported that a patient with AML that may have developed from MDS carried a germline CEBPA mutation: C.134dupC. The germline CEBPA mutation was an initial event in this case, and the patient presented with myelodysplasticity, indicating that this mutation may participate in the transition from MDS to AML.

It has become increasingly clear that familial AML with germline CEBPA mutation involves the inheritance of a single copy of mutated CEBPA encoding a granulocyte differentiation factor (4). The AML is associated with biallelic CEBPA mutations, typically with the germline mutation at the 5 -end of the gene and a somatic mutation at the 3 '-end of the allele acquired when progression to AML occurs (13).

DDX41. DDX41 is a receptor belonging to the DEAD/H-box helicase family, encoded by a gene comprising 17 exons on chromosome 5 (5q35.3) (15). DEAD-box proteins such as DDX41 have a core consisting of two major domains involved in nucleotide binding, with sites for RNA binding and ATP hydrolysis. The functions of the $\mathrm{N}$ - and $\mathrm{C}$-terminal regions are not specific to particular proteins. In terms of RNA metabolism, DDX41 takes part in pre-mRNA splicing, mRNA export, transcriptional and translational regulation, ribosome biogenesis and RNA decay (16).

In multiple families with MDS/AML, DDX41 mutations have been identified as germline and acquired somatic mutations, and most of the germline mutations are frameshift mutations. DDX41 mutations can affect the development of tumors, as the loss of DDX41 has been shown to result in a loss of tumor-suppressive function $(17,18)$. Somatic mutations have also been detected in the majority of tumors with germline mutations (19). Clinically, DDX41 mutation leads to the development of high-risk MDS. Notably, the functions of DDX41 contribute to various biological processes, including mRNA splicing, innate immunity and rRNA processing (15).

In 2015, Polprasert et al (17) first described adult cases of MDS/AML caused by germline mutations (p.I396T, p.F183I, p.Q52fs and p.M155I) in the DDX41 gene. The authors identified a higher incidence of DDX41 mutations and deletions in 
patients with advanced MDS (19\%) compared with low-risk MDS (6\%). They also observed that patients with DDX41 mutations or deletions had poorer overall survival, In addition, they noted that the occurrence of somatic DDX41 mutation is closely associated with the existence of germline DDX41 mutations, with $\sim 50 \%$ of patients with germline DDX41 mutations also acquiring somatic mutations (p.A225D, p.E247K, p.P321L and split-donor site mutations), compared with $0.8 \%$ of patients with wild-type DDX41.

Lewinsohn et al (18) also provided evidence that DDX41 mutations are an important cause of MDS/AML induction, suggesting that DDX41 is an effective tumor suppressor. The authors screened 289 families with hematological malignancies by whole-exon sequencing, and detected heterozygous germline DDX41 mutations in nine families. Three of these families carried a p.D140gfs $* 2$ repeat mutation, one family carried a germline c.1574G $>$ A p.R525H mutation, and five carried new mutations that had not been reported before. In addition, the average age of the germline DDX41 mutation carriers at the onset of MDS or AML was 57 years, which is younger than the previously reported age of 67 years. Further germline DDX41 mutations have been reported in subsequent studies $(19,20)$, including c.711G $>$ T p.L237F, c.712C $>$ A p.P238T, c.155dupA, c.1586_1587delCA and c.719delTinsCG.

Patients with DDX41 mutations who develop MDS/AML usually present with leukopenia with or without other cytopenias and macrocytosis, in addition to hypocellular bone marrow with prominent erythroid dysplasia, and a normal karyotype, often leading to erythroleukemia (18). The prognosis of these patients is generally poor. On the basis of findings in a limited number of patients, cases with DDX41 mutation may respond to lenalidomide (15). However, more data are required to verify the efficacy of this treatment.

RUNX1. RUNX1 is located on chromosome band 21q22, and is associated with the development of normal hematopoiesis. It is a key regulator of hematopoietic and bone marrow differentiation. The protein is a member of a family of transcription factors with a homologous region called the runt homology domain (RHD). The RHD directs the binding of RUNX1 to the DNA sequence of the target gene and mediates the interaction between RUNX1 and core binding factor- $\beta$. Mutations in RUNX1 are associated with leukemia and MDS, and patients with these mutations often have a favorable outcome (21).

In 1999, Osato et al (22) first revealed three types of mutations within the Runt domain of the RUNX1 gene, namely silent mutations, missense mutations and nonsense or frameshift mutations. These mutations can affect the function of RUNX1, leading to leukemia. Since then, additional RUNX1 mutants have been identified in patients with MDS/AML, indicating that germline RUNX1 mutation is one of the main pathogenic mechanisms of MDS and AML. RUNX1 mutations can inhibit the differentiation of HSCs and the onset and development of MDS/AML (22). Ismael et al (23) found RUNX1 mutations, including V90-K117del, Val117fsX124 and T300fsX311, in three of five pediatric patients diagnosed with MDS/MPN-unclassified.

Owen et al (24) conducted a study of familial platelet disease with propensity to myeloid malignancies (FPD/AML), an autosomal dominant syndrome characterized by platelet abnormalities and susceptibility to MDS/AML that is caused by the genetic mutation of RUNX1. The authors identified germline RUNX1 mutations in five families with a history of MDS/AML, including 1007_1013del, G336fsX563, 83insG and A28fsX109, and found a 35\% incidence of MDS/AML in carriers of RUNX1 mutations. In another study of FPD/AML, Cavalcante de Andrade Silva et al (25) examined two brothers who had been diagnosed with hematological malignancies and their families. The study revealed a microdeletion encompassing exons 1-2 of RUNX1 in six family members.

Harada and Harada (26) proposed a new category of myelodysplastic neoplasms, comprising MDS refractory anemia with excess blasts and AML with myelodysplasia-related changes, and sought to elucidate the relationship between RUNX1 mutations and secondary MDS and AML. In their analysis, $20 \%$ of patients with this new category of disease were found to have RUNX1 mutations. The study concluded that RUNX1 mutations are likely to be an initial factor in the development of AML, in addition to other genetic abnormalities.

Different families with germline RUNX1 mutations exhibit varying risks of developing MDS and AML (11-100\%), and the median age of patients with such mutations at the onset of MDS/AML is 33 years, which is younger than that of sporadic MDS/AML $(2,27)$.

GATA2. The GATA2 gene, located on chromosome 3, encodes a zinc finger transcription factor that contains two zinc fingers and a nuclear localization signal. This protein is vital in the development and proliferation of hematopoietic and endocrine cell lineages (28). GATA2 is crucial for the production and maintenance of HSCs in embryonic and adult hematopoietic processes and plays a regulatory role by combining with downstream targets, including transcription factor PU.1 (SPI1), LIM domain only 2 , T-cell acute lymphocytic leukemia protein 1 , Friend leukemia integration 1 transcription factor and RUNX1 (3). Hematopoietic cells are susceptible to changes in GATA2 levels (3). Mutations in the exons and intron 5 of this gene have been identified to cause several hematopoietic diseases, including MDS, AML and chronic myelomonocytic leukemia (CMML). In humans, germline mutations are an important cause of GATA2 deficiency.

While MDS primarily affects the elderly, it is often associated with an underlying genetic predisposition in children and young adults. An et al (29) found that germline GATA2 mutations accounted for $8.5 \%$ of primary MDS cases in a cohort of children in China. Wang et al (30) investigated the prevalence of GATA2 mutations in pediatric hematological diseases related to MDS and AML. Using Sanger sequencing to analyze all exons and intron 5 of GATA2 in children with MDS from three families with recorded pathogenic mutations, they detected GATA2 germline mutations in all three families (c.892dupT p.C298LfsX86, c.1168_1170delAAG p.K390del, c.802G $>$ T p.G268X). In the follow-up sequencing of target genes in six familial MDS patients, GATA2 germline mutation was also found. In two consecutive prospective studies conducted in Germany over 15 years, Wlodarski et al (31) investigated 426 child and adolescent patients with MDS and a further 82 patients with secondary MDS. It was found that GATA2 germline mutations accounted for $15 \%$ of advanced 
primary MDS cases and 7\% of all cases. However, in children with MDS secondary to treatment or acquired aplastic anemia, these mutations were not present. Carriers of GATA2 mutations were older than patients with wild-type GATA1 when diagnosed and were more likely to have monosomy 7 and advanced disease. A further 108 patients with primary MDS were then subjected to a stratified analysis. Among these patients, a total of 57 cases with GATA2 mutations were identified, with 44 different germline mutations, 31 of which were new. It was observed that GATA2 mutations had a prevalence of $37 \%$ in patients of all age groups with monosomy 7 , and peaked at puberty when they were present in $72 \%$ of adolescents with monosomy 7 . The study identified GATA2 mutation as the most common germline defect, with monosomy 7 predisposing adolescents to infantile MDS and a high prevalence of GATA2 mutations.

McReynolds et al (3) conducted a clinical, hematological and genetic evaluation of 25 patients with GATA2 mutations, including missense $(11 / 24,46 \%)$, transcoding $(2 / 24,8 \%)$, acquisition $(5 / 24,21 \%)$ and regulatory $(6 / 24,25 \%)$ mutations. Hematological analyses revealed that $48 \%$ of the patients met the criteria for the diagnosis of MDS or CMML. The study also suggested that abnormal clonal hematopoiesis is frequent in MDS patients with symptomatic GATA2 mutation, indicating the importance of the close monitoring of disease progression in early MDS.

Bödör et al (32) investigated familial cases of MDS/AML with GATA2 germline mutations and observed a GATA2 p.Thr354Met mutation in five individuals from one family pedigree. Furthermore, high-risk MDS syndrome with monosomy 7 was noted in two first cousins with somatic ASXL1 c.1934dupG p.Gly646TrpfsX12. These findings confirm that individuals with germline GATA2 mutations are prone to familial MDS/AML, and the occurrence of monosomy 7 and ASXL1 mutation may be prevalent secondary genetic abnormalities.

Ding et al (28) sequenced the whole genome and exons of a high-risk MDS family comprising an affected father and son, and healthy daughter. On the basis of sequencing results, the affected family members were diagnosed with monocytopenia and mycobacterial infection (MonoMAC) syndrome with a heritable germline GATA2 mutation (R396Q) as a risk factor. Mutations in stromal antigen 2 and ryanodine receptor 2 were also detected in bone marrow samples of the father and son. However, the mutations occurred at different locations, suggesting that these mutations were independently acquired.

Pasquet et al (33) described the preliminary identification of a GATA2 mutation (R396Q) in a mother and her three children by exon sequencing; the mutation was associated with a history of chronic mild neutropenia that developed into AML or MDS. Ten patients with severe chronic neutropenia from six different families were subsequently identified as having six distinct and previously unreported GATA2 mutations (R204X, E224X, R330X, A372T, M388V and complete deletion of the GATA2 locus). The frequent occurrence of MDS and AML in these patients with chronic neutropenia suggests that it is important to screen for GATA2 mutations in chronic neutropenia. The suggestion that GATA2 mutation contributes to the initiation and progression of MDS/AML was supported by Hahn et al (34), who identified a compound in-cis
GATA2 germline mutation in a pedigree with MDS/AML and thrombocytopenia.

GATA2-associated disorders include familial MDS/AML, chronic myeloid leukemia, MonoMAC syndrome and dendritic cell, monocyte, B and NK lymphoid deficiency (30).

Janus kinase 2 (JAK2). The JAK2 gene is located on chromosome 9p. It encodes a non-receptor tyrosine kinase associated with a variety of tumors, particularly hematologic neoplasms. This enzyme is involved in numerous important biological processes, including cell proliferation, differentiation, apoptosis and immune regulation, via participation in the JAK-STAT pathway. The well-known mutation JAK2-V617F is strongly associated with MPNs, including polycythemia vera (PV), essential thrombocythemia (ET) and primary myelofibrosis (PMF) (35).

PMF carries a risk of developing into secondary AML, and mutations of JAK2 have been shown to initiate this progression. Engle et al (36) performed an in-depth sequencing analysis of 649 validated somatic single-nucleotide variants in a single patient at different disease stages. The results indicated that a clonal group comprising JAK2 and U2 small nuclear RNA auxiliary factor 1 represented the founding clone, and included mutations present at high frequencies in all three disease stages.

The majority of mutations in ET are somatic; however, Yoshimitsu et al (37) reported the first case of ET caused by JAK2-T875N mutation in a patient with a family history of thrombocytosis and cerebral infarction, which may have been associated with germline mutations. Marty et al (38) identified two families with germline mutations that led to ET. One family had the JAK2 R867Q mutation, and the other presented with two JAK2 mutations, S755R and R938Q. These unusual mutations exhibited lower sensitivity to JAK2 and HSP90 inhibitors than the JAK2 V617F mutation. Another germline mutation, JAK2 R564Q, was reported by Etheridge et al (39). The authors found that although JAK2 R564Q shows similar levels of increased kinase activity to the JAK2 V617F mutation, the growth-promoting effects of JAK2 R564Q are much milder than those of JAK2 V617F. Although these mutations are of the same gene, their differing drug sensitivities and disease action mechanisms suggests that they affect different functions of the same gene. These distinctions may be associated with the site of the mutation.

ETS variant transcription factor 6 (ETV6). The ETV6 gene is located on chromosome 12. It encodes an ETS family transcription factor with three functional domains necessary for hematopoiesis and the vascular development. Mutations of this gene indicate a predisposition to MDS and acute leukemia, including AML and B-cell acute lymphoblastic leukemia (B-ALL). Patients with ETV6 mutations appear to have decreased platelet numbers with normal-sized platelets and a mild-to-moderate bleeding tendency (40). Several studies have described germline ETV6 variations as a susceptibility factor for hematologic malignancies.

Melazzini et al (41) described ETV6 mutations (P214L, R369W, W380R and N385VFs) leading to ALL or PV. The average platelet counts of these patients were mild to low. The patients were diagnosed at a younger age than those 
without such mutations, with the exception of PV. Similar observations were also made in another studies, in which the ETV6 mutations included R359X, L349P, L358M, R399C and R369Q (40,42-45). Among the carriers of these ETV6 mutations, patients with L349P, N385VFs, P214L and R399C presented with features of MDS. Thrombocytopenia was also observed as a common feature in these patients, but some of the germline mutations were associated with a low platelet count without hematopoietic malignancies. To identify whether ETV6 mutations contribute to childhood leukemia, Topka et al (44) screened a cohort of 588 patients with leukemia. Nineteen distinct ETV6 variants were identified, including two rare germline variants (V37M and R181H).

Lymphocyte adapter protein (LNK)/SH2B adapter protein 3 (SH2B3). The LNK gene, which is also known as SH2B3 and insulin-dependent diabetes mellitus 20 , is located on chromosome 12 , and encodes a lymphocyte adaptor protein. This gene is a negative regulator of multiple cytokine signaling molecules and tyrosine kinase receptors; notably, the lymphocyte adaptor protein acts as a negative regulator of the mutant protein in MPNs with JAK2-V617F mutations (46).

Mutations of LNK/SH2B3 are often present in exon 2, and disturb the normal homeostasis process of HSCs (47-49). Although the majority of LNK variants in MPNs are somatic, germline mutations are also present in sporadic cases, indicating that germline predisposition, including single-nucleotide polymorphisms and haplotypes, may strongly affect the occurrence of MPNs, particularly during leukemic transformation and in idiopathic erythrocytosis (50).

Luque Paz et al (50) reported the case of an 80-year-old man who presented with chronic thrombocytosis. Using next-generation sequencing (NGS), an LNK mutation (c.639C >A p.Ser213Arg) in the PH domain was detected, a mutation that was also reported by Spolverini et al (51).

A germline LNK-E208Q mutation has also been reported $(46,52,53)$. An LNK-E208Q mutation in the $\mathrm{PH}$ domain was detected in five members of a family, two of whom were diagnosed with MPNs. However, this mutation showed low capacity for the promotion of autonomous progenitor cell proliferation, which may explain why the other three family members with this variant did not develop MPN (52).

Oh et al (46) studied 33 samples from patients with JAK2 V617F-negative MPNs and identified two novel mutations in exon 2 of LNK (603_607delGCGCT and 613C>G). These two mutations were identified from DNA in the skin, so it was deduced that both variants were germline mutations. Germline LNK mutations were also detected in a further study of 341 patients with hematopoietic malignancies, including a 1-bp deletion that led to a frameshift and premature stop codon (Q72fs) in a patient with CMML, and a missense mutation (S186I) in four patients, one with PMF and three with CMML (54).

Autophagy related $2 B(A T G 2 B)$ and $G S K 3 B$ interacting protein $(G S K I P)$. Both ATG2B and GSKIP are located on chromosome 14q32. They play a synergistic role in megakaryopoiesis (55). Hematopoietic malignancies caused by ATG2B and GSKIP may be associated with a duplication in $14 q 32$. Plo et al (56) identified a 700-kb tandem duplication at the 14q32.13-q32.2 locus in four families from the West Indies. More than 30 members of these families presented with AML, MDS, CMML or MPN. This locus contains five protein-coding genes: TCL1 family AKT coactivator A, GSKIP, ATG2B, and bradykinin receptors B1 and B2. The researchers found that simultaneous downregulation of ATG2B and GSKIP in this locus led to the reduction of the spontaneous growth of megakaryocytic progenitors, and thereby revealed a novel predisposition locus. However, the opposite conclusion was proposed by Babushok et al (55), in a study of a North American family with an autosomal dominant predisposition to myeloid neoplasms. A duplication of chromosome 14q32 without duplication of GSKIP and ATG2B was detected. Presumably, the duplication in this region may affect the expression of GSKIP and ATG2B. However, whether the duplication of ATG2B and GSKIP is necessary for myeloid neoplasms remains to be determined.

$R B$ binding protein 6 , ubiquitin ligase (RBBP6). The human RBBP6 gene is located on chromosome 16p12.2, and influences cell proliferation and apoptosis by interacting with $\mathrm{p} 53$ and $\mathrm{pRb}$. The RBBP6 gene encodes three proteins: Isoforms 1 and 2 bind the tumor-suppressive proteins p53 and pRb via a RING domain $(57,58)$. Although isoform 3 has no named domain, it may be a cell cycle regulator involved in mitosis and apoptosis (58). Notably, mutations on RBBP6 isoform 3 have shown to be a predisposing factor in several cancers, including lung cancer, breast cancer and MPNs $(57,59)$. Harutyunyan et al (60) detected germline RBBP6 mutations in $\sim 5 \%$ of familial cases of MPN (3/67) and $\sim 0.6 \%$ of sporadic cases (3/490). In familial MPN, they found five mutations on this gene, namely E1654G, R1451T, R1569H, S1444E and A1673V, suggesting that RBBP6 mutations are strong candidates for familial predisposition to MPN.

Other mutations. In recent decades, with the application of advanced biotechnology approaches, an increasing number of germline mutations have been discovered. For example, Narumi et al (61) revealed the loss of chromosome 7 carrying sterile $\alpha$ motif domain-containing 9 germline mutations in two patients with MDS by exome sequencing and other methods. Noris et al (62) found that mutations in 5'-untranslated region of ankyrin repeat domain 26 (ANKRD26) on chromosome $10 \mathrm{p} 12$ may lead to a 30 -fold increase in the incidence of MDS/AML. Takaoka et al (63) reported a new germline helicase-like transcription factor mutation (E259K) in familial MDS. They demonstrated that this mutation may lead to the accumulation of DNA double-strand breaks and the weakening of PCNA polyubiquitination. Both telomerase RNA component (TERC) and telomerase reverse transcriptase (TERT) take part in the assembly of telomerase, which protects chromosomes and stabilizes the genome $(64,65)$. Mutations in TERT or TERC may lead to multiple diseases as presented in Tables I and II. As regard to signal recognition particle 72 (SRP72), limited information is available; however, mutation in this gene is associated with hematological diseases $(64,66)$. Although the existing research on these susceptible genes is relatively superficial, it provides evidence on the pathogenesis of myeloid neoplasms and suggests potential strategies for further diagnosis and treatment. 


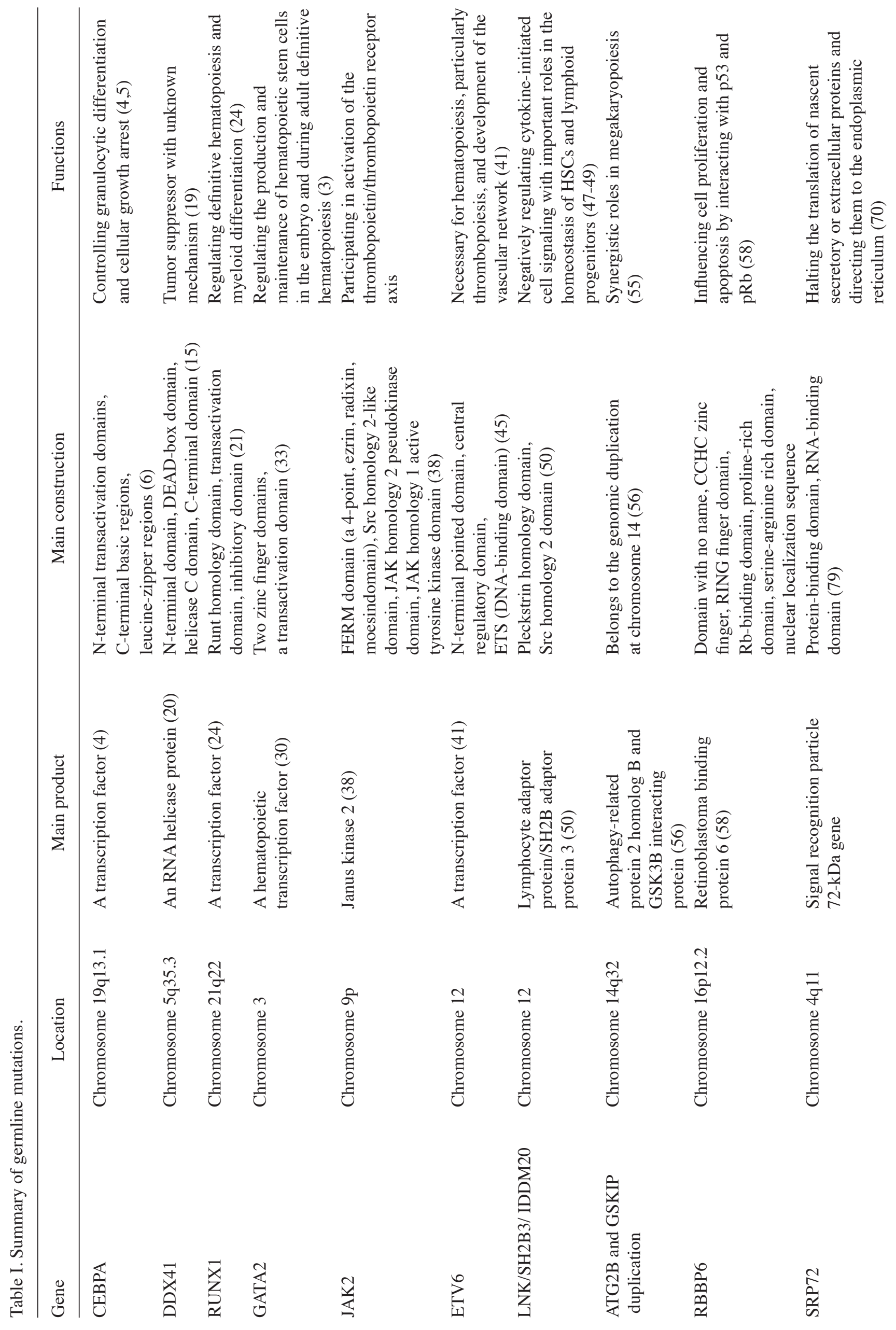




\section{Clinical significance}

Pathogenicity and tumor mutation burden. Pathogenicity may result from the accumulation of various genetic mutations rather than known mutations. In patients with germline mutations, other mutated genes have also been detected $(12,17,30)$. These secondary mutations increase the mutational load, leading to changes in the original disease course and a rapid switch to malignancy $(5,30,67)$. As they accumulate, the pathogenic mechanism appears more complicated. Thus, the detection of gene mutations and mutational burden helps in the analysis and explanation of the pathogenesis and progress of disease from the genetic perspective, and can further improve the level of accurate diagnosis and treatment.

Germline mutations in clinical prediction. Various germline mutations have been identified to be associated with hematological malignancies, but heterogeneity is evident in penetrance, the age of disease onset, clinical manifestations and prognoses. Certain mutations often lead to specific types of hematological tumors appearing in specific clinical syndromes. Mutations at different loci of the same gene may also be a cause of heterogeneity. For example, hereditary MDS is a heterogeneous disease that develops from congenital bone marrow failure syndrome. It seems appropriate that the risk management approach for MDS should differ according to the associated genetic mutations and their diagnoses, including those with current risk such as Fanconi anemia, short-term risk such as RUNX1 mutations, and long-term risk such as DDX41 mutations (68). The following characteristics suggest that genetic susceptibility should be considered in patients with MDS: Physical deformities and dysplasia, recurrent hemopenia, macrocyte and bone marrow failure, repeated infections since childhood, rare types of infections such as Mycobacterium avium infection, multiple concurrent tumors, severe side effects of chemotherapy or radiotherapy, or multiple family members with a family history of malignancy $(69,70)$.

Germline mutations may act as biomarkers for the prediction of drug efficacy. At the 25th European Hematology Association Congress, it was disclosed that patients with DDX41 germline mutations had improved overall survival (HR 0.63, P=0.009) compared with those with wild-type DDX41 when treated with azacytidine. This observation may help physicians to predict the therapeutic response (71).

Germline mutation in treatments. Due to the heterogeneity of myeloid neoplasms, there are no uniform recommendations for treatment. However, HSC transplantation (HSCT) is considered to be an essential treatment for this type of neoplasm. Timely HSCT can avoid the occurrence of primary infections, organ dysfunction and malignancy. Nevertheless, as germline mutations have familial aggregation, family members with germline mutations also have a high risk of acquiring diseases, and the use of HSCT donations from relatives can lead to recurrence (72). Therefore, donors must be screened for potential germline mutations to eliminate the potential risk of donor-derived recurrence. Moreover, genetic consulting and testing is also recommended for other family members (73). Abnormal bone marrow is a good predictor of prognosis, and 
Table II. Germline mutation-associated syndromes or diseases in myeloid neoplasms.

\begin{tabular}{|c|c|}
\hline Genes & Associated syndromes or diseases \\
\hline CEBPA & $\begin{array}{l}\text { Familial AML, particularly FAB M1, M1Eo, } \\
\text { M2 and M2Eo (11-13), MDS (11-13) }\end{array}$ \\
\hline DDX41 & $\begin{array}{l}\text { Familial MDS/AML, CML, non-Hodgkin lymphoma, } \\
\text { Hodgkin lymphoma (18) }\end{array}$ \\
\hline RUNX1 & $\begin{array}{l}\text { Familial platelet disease/AML, MDS/AML, } \\
\text { MPNs (26) }\end{array}$ \\
\hline GATA2 & $\begin{array}{l}\text { Familial MDS/AML, CML, MonoMAC syndrome, } \\
\text { DCML deficiency, Emberger syndrome } \\
\text { (lymphoedema, monosomy } 7 \text { and MDS) (30) }\end{array}$ \\
\hline
\end{tabular}

JAK2 MPNs, including PV, ET and PMF (35), secondary
AML from PMF (36)

ETV6 ETV6-RT inherited thrombocytopenia predisposes to childhood ALL (41), ALL (45), red cell macrocytosis (45)

LNK/SH2B3/ Pediatric B-ALL (48), familial MPNs, including

IDDM20 PV, ET and PMF $(46,52,53)$

ATG2B and Familial MPNs (56)

GSKIP

duplication

RBBP6

Familial MPNs

SRP72

Familial BMF syndrome with elevated risk of MDS/AML $(64,66)$

TERC Dyskeratosis congenita, BMF syndrome with elevated risk of AA, MDS and AML $(65,66,80), \mathrm{AA}(81-83)$, AML $(82,84)$, MDS (82-84), paroxysmal nocturnal hemoglobulinemia (83)

TERT
Dyskeratosis congenita, BMF syndrome with elevated risk of AA, MDS and AML $(65,66,80)$, MPNs including PV, ET, PMF (87-89), AML $(90,91)$, AA (92), MDS evolving into AML (86)
Early age of AML onset $(11,12)$; favorable outcomes in patients with AML (11-13)

Late age of MDS/AML onset $(17,18)$; inferior overall survival (17); improved response to lenalidomide (17) Outcomes for MDS/AML are heterogeneous and effective treatment options are limited (24)

Early age of MDS onset with a high risk of AML (29); heterogeneous manifestations in multiple systems; GATA2 mutational status does not negatively affect the outcome of MDS or HSCT (31)

JAK2 V617F is a canonical mutation in MPN (37); different mutations of JAK2 vary in drug sensitivity

Thrombocytopenia; early leukemic transformation is a major risk (41); children with ALL with germline ETV6 variants are significantly older (42)

High SH2B3 levels are associated with longer event-free survival and overall survival in pediatric patients with B-ALL (48)

Unclear whether the duplications of the GSKIP and ATG2B genes are necessary for familial myeloid neoplasms $(55,56)$

Clinical features and survival of familial MPNs are similar to those of sporadic MPN (59)

Little known due to limited cases $(64,66)$

TERC mutations disturb telomerase functions, leading to dyskeratosis congenita, BMF and MPNs and a high risk of AA/MDS/AML; highly variable clinical phenotypes from mild to severe $(64,85)$; patients with AA and TERC mutations have an inadequate response to immunosuppression (86) and exhibit worse survival (65)

TERT mutations disturb telomerase functions, leading to dyskeratosis congenita, BMF and MPNs and a high risk of AA/MDS/AML; highly variable clinical pheno typesfrom mild to severe $(64,85)$; patients with AA and TERT mutations have an inadequate response to immunosuppression (86) and exhibit worse survival (65); in AML, the highest median TERT levels are in M1 and M7, and higher TERT levels indicate a significantly lower overall survival in M1 (90)

CEBPA, CCAAT enhancer binding protein $\alpha$; DDX41, DEAD (Asp-Glu-Ala-Asp) box polypeptide 41; RUNX1, RUNX family transcription factor 1; GATA2, GATA binding protein 2; JAK2, Janus kinase 2; ETV6, ETS variant transcription factor 6; LNK, lymphocyte adapter protein; SH2B3, SH2B adapter protein 3; IDDM20, insulin-dependent diabetes mellitus 20; ATG2B, autophagy related 2B; GSKIP, GSK3B interacting protein; RBBP6, RB binding protein 6, ubiquitin ligase; SRP72, signal recognition particle 72; TERC, telomerase RNA component; TERT, telomerase reverse transcriptase; AML, acute myeloid leukemia; FAB, French-American-British classification; M1Eo, M1 with eosinophilia; M2Eo, M2 with eosinophilia; MDS, myelodysplastic syndrome; CML, chronic myeloid leukemia; MPNs, myeloproliferative neoplasms; MonoMAC, monocytopenia and mycobacterial infection; DCML, dendritic cell, monocyte, B and NK lymphoid; PV, polycythemia vera; ET, essential thrombocythemia; PMF, primary myelofibrosis; ALL, acute lymphoblastic leukemia; B-ALL, B-cell ALL; AA, aplastic anemia; BMF, bone marrow failure. 
not all mutations indicate the need for HSCT, so monitoring is essential.

Targeted therapy is also a topic of great interest in cancer therapy. Potential targets associated with germline mutations include the mutated gene itself, the products expressed and the pathway involved. Germline mutations result in diseases by influencing the bone marrow microenvironment. It has been reported that leukemias associated with germline mutations cannot be securely managed with HSCT alone; it is also necessary to treat the aberrant microenvironment, as therapy targeting abnormal chemokine production from mutated genes may help reverse the process of leukemia (74).

Gene therapy has potential as an future treatment. The introduction of normal genes into reproductive cells, i.e., sperm and eggs, or preimplantation embryos by germline gene editing can provide favorable outcomes for patients and prevent the passage of genetic disease to future generations (75). The key to this therapy is the choice of genetic vectors and the effective expression of the genes inserted into them. Frequently used vectors of choice include those based on adeno-associated viruses, retroviruses adenoviruses and herpes viruses. Effective expression is essential for the therapeutic level of the protein to be reached. However, at present, this technology is only being researched in mice and human preimplantation embryos so that its safety and efficacy may be studied and for ethical reasons.

Somatic mutation, gene modification and germline mutation. Germline mutations may change the structures of genes, which can lead to the acquisition of somatic mutations in the same genes, with germline mutations acting as an initial trigger $(30,67)$. The somatic mutation is often identified as a second hit and accelerates the disease process by disabling the function of the gene and enhancing the clonal advantages of the germline mutation making the disease more complex. These two types of mutations imply different prognoses. Medullary tumors with DDX41 mutations were reported to have a good prognosis. Moreover, the overall response rate (ORR) to hypomethylating drugs in patients with germline and somatic mutations was $63 \%$, while the ORR of patients with only somatic mutations was $75 \%$. Therefore, it may be concluded that patients who have medullary tumors with DDX41 somatic mutations have a good prognosis and may be considered as an independent population (76). By sequencing samples from the oral mucosa, saliva, fingernails or hair follicles, these individuals may be identified and further clinical measures taken.

Along with genetic mutations, genetic modifications can occur. Variations, which mainly include DNA hypermethylation, histone modifications and changes to non-coding RNAs, play a critical role in the pathogenesis of myeloid neoplasms by inhibiting tumor suppressor genes or increasing the expression of oncogenes $(77,78)$. Drugs targeting DNA methylation and histone deacetylation enzymes are associated with an improved outcome. The use of azacitidine is beneficial for high-risk MDS patients, as it reduces the remission rate, controls the disease and prolongs survival (71). Histone deacetylase inhibitors are an emerging class of drug, as an alternative to hypomethylating agents (HMAs). As MDS is only mildly responsive to HMAs with low CR and PR rate
(20-35\%) when used as monotherapy (78), genetic modifications may also act as a prognostic biomarker.

\section{Summary and prospects}

The revision of the myeloid tumor classification guidelines by the WHO in 2016 highlighted the importance of germline susceptibility genes, by creating a category of myeloid tumors with germline susceptibility. In the past few years, the number of susceptibility genes discovered by NGS technology has increased sharply, and includes GATA2, ANKRD26, ETV6, SRP72, DDX41, TERC and TERT. Moreover, most germline mutations are accompanied by somatic mutations, which may be an important reason for secondary genetic abnormalities. The discovery of susceptibility genes reveals the possible pathogenesis of myeloid neoplasms from the perspective of molecular biology. This research avenue provides further directions for clinical diagnosis, drug application and even gene therapy. However, due to differences among samples and the pathological complexity of myeloid neoplasms, genetic predisposition research is relatively scattered and cannot be accurately classified. Nevertheless, as research into this subject deepens, it is likely that scientists will find effective treatments for this intractable disease.

\section{Acknowledgements}

Not applicable.

\section{Funding}

The present study was supported by a grant from the National Natural Science Foundation of China (General Program, grant no. 81870103).

\section{Availability of data and materials}

Not applicable.

\section{Authors' contributions}

$\mathrm{LB}$ and BD were responsible for conceptualization of the study. LW, ZL and BF curated the data and LB, TM and XL analyzed the data. BD supervised the study and acquired funding. XC contributed to conceptualization, supervised the study and reviewed the manuscript. $\mathrm{LB}$ wrote the original draft of the manuscript, and LB and BD reviewed and edited the manuscript. All authors read and approved the final manuscript.

\section{Ethics approval and consent to participate}

Not applicable.

\section{Patient consent for publication}

Not applicable.

\section{Competing interests}

The authors declare that they have no competing interests. 


\section{References}

1. Arber DA, Orazi A, Hasserjian R, Thiele J, Borowitz MJ Le Beau MM, Bloomfield CD, Cazzola M and Vardiman JW: The 2016 revision to the World Health Organization classification of myeloid neoplasms and acute leukemia. Blood 127: 2391-2405, 2016.

2. Geyer JT: Myeloid neoplasms with germline predisposition. Pathobiology 86: 53-61, 2019.

3. McReynolds LJ, Yang Y, Yuen Wong H, Tang J, Zhang Y, Mulé MP, Daub J, Palmer C, Foruraghi L, Liu Q, et al: MDS-associated mutations in germline GATA2 mutated patients with hematologic manifestations. Leuk Res 76: 70-75, 2019.

4. Wen XM, Hu JB, Yang J, Qian W, Yao DM, Deng ZQ, Zhang YY, Zhu XW, Guo H, Lin J and Qian J: CEBPA methylation and mutation in myelodysplastic syndrome. Med Oncol 32: 192, 2015

5. Tawana K, Wang J, Renneville A, Bödör C, Hills R, Loveday C, Savic A, Van Delft FW, Treleaven J, Georgiades P, et al: Disease evolution and outcomes in familial AML with germline CEBPA mutations. Blood 126: 1214-1223, 2015.

6. Smith ML, Cavenagh JD, Lister TA and Fitzgibbon J: Mutation of CEBPA in familial acute myeloid leukemia. N Engl J Med 351: 2403-2407, 2004.

7. Cammenga J, Mulloy JC, Berguido FJ, MacGrogan D, Viale A and Nimer SD: Induction of C/EBPalpha activity alters gene expression and differentiation of human CD34+ cells. Blood 101: 2206-2214, 2003.

8. Zhang P, Iwasaki-Arai J, Iwasaki H, Fenyus ML, Dayaram T, Owens BM, Shigematsu H, Levantini E, Huettner CS, Lekstrom-Himes JA, et al: Enhancement of hematopoietic stem cell repopulating capacity and self-renewal in the absence of the transcription factor C/EBP alpha. Immunity 21: 853-863, 2004.

9. Schwieger M, Löhler J, Fischer M, Herwig U, Tenen DG and Stocking C: A dominant-negative mutant of C/EBPalpha, associated with acute myeloid leukemias, inhibits differentiation of myeloid and erythroid progenitors of man but not mouse. Blood 103: 2744-2752, 2004.

10. Sellick GS, Spendlove HE, Catovsky D, Pritchard-Jones K and Houlston RS: Further evidence that germline CEBPA mutations cause dominant inheritance of acute myeloid leukaemia. Leukemia 19: 1276-1278, 2005.

11. Nanri T, Uike N, Kawakita T, Iwanaga E, Mitsuya H and Asou N: A family harboring a germ-line $\mathrm{N}$-terminal C/EBPalpha mutation and development of acute myeloid leukemia with an additional somatic C-terminal C/EBPalpha mutation. Genes Chromosomes Cancer 49: 237-241, 2010.

12. Pabst T, Eyholzer M, Haefliger S, Schardt J and Mueller BU: Somatic CEBPA mutations are a frequent second event in families with germline CEBPA mutations and familial acute myeloid leukemia. J Clin Oncol 26: 5088-5093, 2008

13. Renneville A, Mialou V, Philippe N, Kagialis-Girard S, Biggio V, Zabot MT, Thomas X, Bertrand Y and Preudhomme C: Another pedigree with familial acute myeloid leukemia and germline CEBPA mutation. Leukemia 23: 804-806, 2009.

14. Yan B, Ng C, Moshi G, Ban K, Lee PL, Seah E, Chiu L, Koay ES, Liu TC, Ng CH, et al: Myelodysplastic features in a patient with germline CEBPA-mutant acute myeloid leukaemia. J Clin Pathol 69: 652-654, 2016.

15. Cheah JJC, Hahn CN, Hiwase DK, Scott HS and Brown AL: Myeloid neoplasms with germline DDX41 mutation. Int J Hematol 106: 163-174, 2017.

16. Jiang Y, Zhu Y, Qiu W, Liu YJ, Cheng G, Liu ZJ and Ouyang S: Structural and functional analyses of human DDX41 DEAD domain. Protein Cell 8: 72-76, 2017.

17. Polprasert C, Schulze I, Sekeres MA, Makishima H, Przychodzen B, Hosono N, Singh J, Padgett RA, Gu X, Phillips JG, et al: Inherited and somatic defects in DDX41 in myeloid neoplasms. Cancer Cell 27: 658-670, 2015.

18. Lewinsohn M, Brown AL, Weinel LM, Phung C, Rafidi G, Lee MK, Schreiber AW, Feng J, Babic M, Chong CE, et al: Novel germ line DDX41 mutations define families with a lower age of MDS/AML onset and lymphoid malignancies. Blood 127: 1017-1023, 2016

19. Cardoso SR, Ryan G, Walne AJ, Ellison A, Lowe R, Tummala H, Rio-Machin A, Collopy L, Al Seraihi A, Wallis Y, et al: Germline heterozygous DDX41 variants in a subset of familial myelodysplasia and acute myeloid leukemia. Leukemia 30 2083-2086, 2016.
20. Li R, Sobreira N, Witmer PD, Pratz KW and Braunstein EM: Two novel germline DDX41 mutations in a family with inherited myelodysplasia/acute myeloid leukemia. Haematologica 101: e228-e231, 2016.

21. Al-Harbi S, Aljurf M, Mohty M, Almohareb F and Ahmed SOA: An update on the molecular pathogenesis and potential therapeutic targeting of AML with t(8;21)(q22;q22.1);RUNX1-RUNX1T1. Blood Adv 4: 229-238, 2020.

22. Osato M, Asou N, Abdalla E, Hoshino K, Yamasaki H, Okubo T, Suzushima H, Takatsuki K, Kanno T, Shigesada K and Ito Y: Biallelic and heterozygous point mutations in the runt domain of the AML1/PEBP2alphaB gene associated with myeloblastic leukemias. Blood 93: 1817-1824, 1999.

23. Ismael O, Shimada A, Hama A, Elshazley M, Muramatsu H, Goto A, Sakaguchi H, Tanaka M, Takahashi Y, Yinyan X, et al: De novo childhood myelodysplastic/myeloproliferative disease with unique molecular characteristics. Brit J Haematol 158: 129-137, 2012.

24. Owen CJ, Toze CL, Koochin A, Forrest DL, Smith CA, Stevens JM, Jackson SC, Poon M, Sinclair GD, Leber B, et al: Five new pedigrees with inherited RUNX1 mutations causing familial platelet disorder with propensity to myeloid malignancy. Blood 112: 4639-4645, 2008.

25. Cavalcante de Andrade Silva M, Krepischi ACV, Kulikowski LD, Zanardo EA, Nardinelli L, Leal AM, Costa SS, Muto NH, Rocha V and Velloso EDRP: Deletion of RUNX1 exons 1 and 2 associated with familial platelet disorder with propensity to acute myeloid leukemia. Cancer Genet 222-223: 32-37, 2018.

26. Harada $\mathrm{Y}$ and Harada $\mathrm{H}$ : Molecular mechanisms that produce secondary MDS/AML by RUNX1/AML1 point mutations. J Cell Biochem 112: 425-432, 2011

27. Galera P, Dulau-Florea A and Calvo KR: Inherited thrombocytopenia and platelet disorders with germline predisposition to myeloid neoplasia. Int J Lab Hematol 41 (Suppl 1): S131-S141, 2019.

28. Ding LW, Ikezoe T, Tan KT, Mori M, Mayakonda A, Chien W, Lin DC, Jiang YY, Lill M, Yang H, et al: Mutational profiling of a MonoMAC syndrome family with GATA2 deficiency. Leukemia 31: 244-245, 2017.

29. An WB, Liu C, Wan Y, Chen XY, Guo Y, Chen XJ, Yang WY Chen YM, Zhang YC and Zhu XF: Clinical and molecular characteristics of GATA2 related pediatric primary myelodysplastic syndrome. Zhonghua Xue Ye Xue Za Zhi 40: 477-483, 2019 (In Chinese).

30. Wang X, Muramatsu H, Okuno Y, Sakaguchi H, Yoshida K, Kawashima N, Xu Y, Shiraishi Y, Chiba K, Tanaka H, et al: GATA2 and secondary mutations in familial myelodysplastic syndromes and pediatric myeloid malignancies. Haematologica 100: e398-e401, 2015.

31. Wlodarski MW, Hirabayashi S, Pastor V, Starý J, Hasle H, Masetti R, Dworzak M, Schmugge M, van den Heuvel-Eibrink M, Ussowicz M, et al: Prevalence, clinical characteristics, and prognosis of GATA2-related myelodysplastic syndromes in children and adolescents. Blood 127: 1387-1397, 1518, 2016.

32. Bödör C, Renneville A, Smith M, Charazac A, Iqbal S Etancelin $\mathrm{P}$, Cavenagh J, Barnett MJ, Kramarzová K, Krishnan B, et al: Germ-line GATA2 p.THR354MET mutation in familial myelodysplastic syndrome with acquired monosomy 7 and ASXL1 mutation demonstrating rapid onset and poor survival. Haematologica 97: 890-894, 2012.

33. Pasquet M, Bellanné-Chantelot C, Tavitian S, Prade N, Beaupain B, Larochelle O, Petit A, Rohrlich P, Ferrand C, Van Den Neste E, et al: High frequency of GATA2 mutations in patients with mild chronic neutropenia evolving to MonoMac syndrome, myelodysplasia, and acute myeloid leukemia. Blood 121: 822-829, 2013.

34. Hahn CN, Brautigan PJ, Chong CE, Janssan A, Venugopal P, Lee Y, Tims AE, Horwitz MS, Klingler-Hoffmann $M$ and Scott HS: Characterisation of a compound in-cis GATA2 germline mutation in a pedigree presenting with myelodysplastic syndrome/acute myeloid leukemia with concurrent thrombocytopenia. Leukemia 29: 1795-1797, 2015.

35. Kralovics R, Passamonti F, Buser AS, Teo S, Tiedt R, Passweg JR, Tichelli A, Cazzola M and Skoda RC: A gain-of-function mutation of JAK2 in myeloproliferative disorders. N Engl J Med 352: 1779-1790, 2005

36. Engle EK, Fisher DA, Miller CA, McLellan MD, Fulton RS, Moore DM, Wilson RK, Ley TJ and Oh ST: Clonal evolution revealed by whole genome sequencing in a case of primary myelofibrosis transformed to secondary acute myeloid leukemia. Leukemia 29: 869-876, 2015. 
37. Yoshimitsu M, Hachiman M, Uchida Y, Arima N, Arai A Kamada Y, Shide K, Ito M, Shimoda K and Ishitsuka K: Essential thrombocytosis attributed to JAK2-T875N germline mutation. Int J Hematol 110: 584-590, 2019.

38. Marty C, Saint-Martin C, Pecquet C, Grosjean S, Saliba J, Mouton C, Leroy E, Harutyunyan AS, Abgrall JF, Favier R, et al: Germ-line JAK2 mutations in the kinase domain are responsible for hereditary thrombocytosis and are resistant to JAK2 and HSP90 inhibitors. Blood 123: 1372-1383, 2014.

39. Etheridge SL, Cosgrove ME, Sangkhae V, Corbo LM, Roh ME, Seeliger MA, Chan EL and Hitchcock IS: A novel activating, germline JAK2 mutation, JAK2R564Q, causes familial essential thrombocytosis. Blood 123: 1059-1068, 2014.

40. Poggi M, Canault M, Favier M, Turro E, Saultier P, Ghalloussi D, Baccini V, Vidal L, Mezzapesa A, Chelghoum N, et al: Germline variants in ETV6 underlie reduced platelet formation, platelet dysfunction and increased levels of circulating CD34+ progenitors. Haematologica 102: 282-294, 2017.

41. Melazzini F, Palombo F, Balduini A, De Rocco D, Marconi C, Noris P, Gnan C, Pippucci T, Bozzi V, Faleschini M, et al Clinical and pathogenic features of ETV6-related thrombocytopenia with predisposition to acute lymphoblastic leukemia. Haematologica 101: 1333-1342, 2016.

42. Moriyama T, Metzger ML, Wu G, Nishii R, Qian M, Devidas M, Yang W, Cheng C, Cao X, Quinn E, et al: Germline genetic variation in ETV6 and risk of childhood acute lymphoblastic leukaemia: A systematic genetic study. Lancet Oncol 16 1659-1666, 2015

43. Zhang MY, Churpek JE, Keel SB, Walsh T, Lee MK, Loeb KR Gulsuner S, Pritchard CC, Sanchez-Bonilla M, Delrow JJ, et al: Germline ETV6 mutations in familial thrombocytopenia and hematologic malignancy. Nat Genet 47: 180-185, 2015.

44. Topka S, Vijai J, Walsh MF, Jacobs L, Maria A, Villano D, Gaddam P, Wu G, McGee RB, Quinn E, et al: Germline ETV6 mutations confer susceptibility to acute lymphoblastic leukemia and thrombocytopenia. PLoS Genet 11: e1005262, 2015.

45. Noetzli L, Lo RW, Lee-Sherick AB, Callaghan M, Noris $P$ Savoia A, Rajpurkar M, Jones K, Gowan K, Balduini C, et al: Germline mutations in ETV6 are associated with thrombocytopenia, red cell macrocytosis and predisposition to lymphoblastic leukemia. Nat Genet 47: 535-538, 2015.

46. Oh ST, Simonds EF, Jones C, Hale MB, Goltsev Y, Gibbs KD Jr, Merker JD, Zehnder JL, Nolan GP and Gotlib J: Novel mutations in the inhibitory adaptor protein LNK drive JAK-STAT signaling in patients with myeloproliferative neoplasms. Blood 116 988-992, 2010.

47. Maslah N, Cassinat B, Verger E, Kiladjian JJ and Velazquez L: The role of LNK/SH2B3 genetic alterations in myeloproliferative neoplasms and other hematological disorders. Leukemia 31: $1661-1670,2017$

48. Yano M, Imamura T, Asai D, Deguchi T, Hashii Y, Endo M, Sato A, Kawasaki H, Kosaka Y, Kato K, et al: Clinical significance of SH2B3 (LNK) expression in paediatric B-cell precursor acute lymphoblastic leukaemia. Br J Haematol 183: 327-330, 2018.

49. McMullin MF and Cario $\mathrm{H}$ : LNK mutations and myeloproliferative disorders. Am J Hematol 91: 248-251, 2016.

50. Luque Paz D, Boyer F, Beucher A, Bouvier A, Jouanneau-Courville R, Guardiola P, Lambert D, Delneste Y, Hunault M, Blanchet $\mathrm{O}$ and Ugo V: Concomitant CALR and LNK mutations leading to essential thrombocythemia with erythrocytosis. Blood Cells Mol Dis 71: 75-76, 2018.

51. Spolverini A, Pieri L, Guglielmelli P, Pancrazzi A, Fanelli T, Paoli C, Bosi A, Nichele I, Ruggeri M and Vannucchi AM: Infrequent occurrence of mutations in the PH domain of LNK in patients with JAK2 mutation-negative 'idiopathic' erythrocytosis. Haematologica 98: e101-e102, 2013

52. Loscocco GG, Mannarelli C, Pacilli A, Fanelli T, Rotunno G, Gesullo F, Corbizi-Fattori G, Vannucchi AM and Guglielmelli P: Germline transmission of LNKE208Q variant in a family with myeloproliferative neoplasms. Am J Hematol 91: E356, 2016.

53. Rumi E, Harutyunyan AS, Pietra D, Feenstra JD, Cavalloni C Roncoroni E, Casetti I, Bellini M, Milanesi C, Renna MC, et al: LNK mutations in familial myeloproliferative neoplasms. Blood 128: 144-145, 2016

54. Oh ST, Zahn JM, Jones CD, Zhang B, Loh ML, Kantarjian H, Simonds EF, Bruggner RV, Abidi P, Natsoulis G, et al: Identification of novel LNK mutations in patients with chronic myeloproliferative neoplasms and related disorders. Blood 116: 315,2010 .
55. Babushok DV, Stanley NL, Morrissette JJD, Lieberman DB, Olson TS, Chou ST and Hexner EO: Germline duplication of ATG2B and GSKIP genes is not required for the familial myeloid malignancy syndrome associated with the duplication of chromosome 14q32. Leukemia 32: 2720-2723, 2018.

56. Plo I, Bellanné-Chantelot C and Vainchenker W: ATG2B and GSKIP: 2 new genes predisposing to myeloid malignancies. Mol Cell Oncol 3: e1094564, 2015.

57. Hu D, Zhang S, Zhao Y, Wang S, Wang Q, Song X, Lu D, Mao $\mathrm{Y}$ and Chen $\mathrm{H}$ : Association of genetic variants in the retinoblastoma binding protein 6 gene with the risk of glioma: A case-control study in a Chinese Han population. J Neurosurg 121: 1209-1218, 2014

58. Mbita Z, Meyer M, Skepu A, Hosie M, Rees J and Dlamini Z: De-regulation of the RBBP6 isoform 3/DWNN in human cancers. Mol Cell Biochem 362: 249-262, 2012.

59. Rumi E and Cazzola M: Advances in understanding the pathogenesis of familial myeloproliferative neoplasms. Brit J Haematol 178: 689-698, 2017.

60. Harutyunyan AS, Giambruno R, Krendl C, Stukalov A, Klampfl T, Berg T, Chen D, Milosevic Feenstra JD, Jäger R, Gisslinger B, et al: Germline RBBP6 mutations in familial myeloproliferative neoplasms. Blood 127: 362-365, 2016.

61. Narumi S, Amano N, Ishii T, Katsumata N, Muroya K, Adachi M, Toyoshima K, Tanaka Y, Fukuzawa R, Miyako K, et al: SAMD9 mutations cause a novel multisystem disorder, MIRAGE syndrome, and are associated with loss of chromosome 7. Nat Genet 48: 792-797, 2016.

62. Noris P, Perrotta S, Seri M, Pecci A, Gnan C, Loffredo G, Pujol-Moix N, Zecca M, Scognamiglio F, De Rocco D, et al: Mutations in ANKRD26 are responsible for a frequent form of inherited thrombocytopenia: Analysis of 78 patients from 21 families. Blood 117: 6673-6680, 2011.

63. Takaoka K, Kawazu M, Koya J, Yoshimi A, Masamoto Y, Maki H, Toya T, Kobayashi T, Nannya Y, Arai S, et al: A germline HLTF mutation in familial MDS induces DNA damage accumulation through impaired PCNA polyubiquitination. Leukemia 33: 1773-1782, 2019.

64. Duployez N, Lejeune S, Renneville A and Preudhomme C: Myelodysplastic syndromes and acute leukemia with genetic predispositions: A new challenge for hematologists. Expert Rev Hematol 9: 1189-1202, 2016.

65. Townsley DM, Dumitriu B and Young NS: Bone marrow failure and the telomeropathies. Blood 124: 2775-2783, 2014.

66. Király AP, Kállay K, Gángó A, Kellner Á, Egyed M, Szőke A, Kiss R, Vályi-Nagy I, Csomor J, Matolesy A and Bödör C: Familial acute myeloid leukemia and myelodysplasia in hungary. Pathol Oncol Res 24: 83-88, 2018.

67. McReynolds LJ, Zhang Y, Yang Y, Tang J, Mulé M, Hsu AP, Townsley DM, West RR, Zhu J, Hickstein DD, et al: Rapid progression to AML in a patient with germline GATA2 mutation and acquired NRAS Q61K mutation. Leuk Res Rep 12: 100176 , 2019.

68. Rafei $\mathrm{H}$ and DiNardo CD: Hereditary myeloid malignancies. Best Pract Res Clin Haematol 32: 163-176, 2019.

69. Wiggins $M$ and Stevenson W: Genetic predisposition in acute leukaemia. Int J Lab Hematol 42 (Suppl 1): S75-S81, 2020.

70. Babushok DV, Bessler M and Olson TS: Genetic predisposition to myelodysplastic syndrome and acute myeloid leukemia in children and young adults. Leuk Lymphoma 57: 520-536, 2016.

71. Nannya Y, Tobiasson M, Bernard E, Sato S, Creignou M, Takeda J, Anying Z, Shiraishi Y, Chiba K, Tanaka H, et al: Molecular characteristics that predict response to azacitidine therapy. Blood 134 (Suppl 1): 4246, 2019.

72. Galera P, Hsu AP, Wang W, Droll S, Chen R, Schwartz JR, Klco JM, Arai S, Maese L, Zerbe C, et al: Donor-derived MDS/ AML in families with germline GATA2 mutation. Blood 132: 1994-1998, 2018

73. Bochtler T, Haag G, Schott S, Kloor M, Krämer A and Müller-Tidow C: Hematological malignancies in adults with a family predisposition. Dtsch Arztebl Int 115: 848-854, 2018.

74. Miller LH, Qu CK and Pauly M: Germline mutations in the bone marrow microenvironment and dysregulated hematopoiesis. Exp Hematol 66: 17-26, 2018.

75. Wolf DP, Mitalipov PA and Mitalipov SM: Principles of and strategies for germline gene therapy. Nat Med 25: 890-897, 2019.

76. Qu S, Li B, Qin T, Xu Z, Pan L, Hu N, Huang G, Peter Gale R and Xiao Z: Molecular and clinical features of myeloid neoplasms with somatic DDX41 mutations. Br J Haematol: Apr 19, 2020 (Epub ahead of print). 
77. Jones L, McCarthy P and Bond J: Epigenetics of paediatric acute myeloid leukaemia. Brit J Haematol 188: 63-76, 2020.

78. Liu XL, Liu HQ, Li J, Mao CY, He JT and Zhao X: Role of epigenetic in leukemia: From mechanism to therapy. Chem Biol Interact 317: 108963, 2020.

79. Becker MMM, Lapouge K, Segnitz B, Wild K and Sinning I: Structures of human SRP72 complexes provide insights into SRP RNA remodeling and ribosome interaction. Nucleic Acids Res 45: 470-481, 2017.

80. Tamary $\mathrm{H}$ and Alter BP: Current diagnosis of inherited bone marrow failure syndromes. Pediatr Hemat Oncol 24: 87-99, 2007.

81. Balakumaran A, Mishra PJ, Pawelczyk E, Yoshizawa S, Sworder BJ, Cherman N, Kuznetsov SA, Bianco P, Giri N, Savage SA, et al: Bone marrow skeletal stem/progenitor cell defects in dyskeratosis congenita and telomere biology disorders. Blood 125: 793-802, 2015.

82. Marrone A, Sokhal P, Walne A, Beswick R, Kirwan M, Killick S Williams M, Marsh J, Vulliamy T and Dokal I: Functional characterization of novel telomerase RNA (TERC) mutations in patients with diverse clinical and pathological presentations. Haematologica 92: 1013-1020, 2007.

83. Yamaguchi H, Baerlocher GM, Lansdorp PM, Chanock SJ, Nunez O, Sloand E and Young NS: Mutations of the human telomerase RNA gene (TERC) in aplastic anemia and myelodysplastic syndrome. Blood 102: 916-918, 2003.

84. Yan S, Han B, Wu Y, Zhou D and Zhao Y: Telomerase gene mutation screening and telomere overhang detection in Chinese patients with acute myeloid leukemia. Leuk Lymphoma 54: 1437-1441, 2013.

85. Both A, Krauter J, Damm F, Thol F, Göhring G, Heuser M, Ottmann O, Lübbert M, Wattad M, Kanz L, et al: The hypomorphic TERT A1062T variant is associated with increased treatment-related toxicity in acute myeloid leukemia. Ann Hematol 96: 895-904, 2017.

86. Yamaguchi H, Calado RT, Ly H, Kajigaya S, Baerlocher GM, Chanock SJ, Lansdorp PM and Young NS: Mutations in TERT, the gene for telomerase reverse transcriptase, in aplastic anemia. N Engl J Med 352: 1413-1424, 2005.
87. Chiang YH, Chang YC, Lin HC, Huang L, Cheng CC, Wang WT, Cheng HI, Su NW, Chen CG, Lin J, et al: Germline variations at JAK2, TERT, HBS1L-MYB and MECOM and the risk of myeloproliferative neoplasms in Taiwanese population. Oncotarget 8: 76204-76213, 2017.

88. Trifa AP, Bănescu C, Bojan AS, Voina CM, Popa S, Vișan S, Ciubean AD, Tripon F, Dima D, Popov VM, et al: MECOM, HBS1L-MYB, THRB-RARB, JAK2, and TERT polymorphisms defining the genetic predisposition to myeloproliferative neoplasms: A study on 939 patients. Am J Hematol 93: 100-106, 2018.

89. Oddsson A, Kristinsson SY, Helgason H, Gudbjartsson DF, Masson G, Sigurdsson A, Jonasdottir A, Jonasdottir A Steingrimsdottir $\mathrm{H}$, Vidarsson $\mathrm{B}$, et al: The germline sequence variant rs2736100_C in TERT associates with myeloproliferative neoplasms. Leukemia 28: 1371-1374, 2014.

90. Kim HJ, Choi EJ, Sohn HJ, Park SH, Min WS and Kim TG: Combinatorial molecular marker assays of WT1, survivin, and TERT at initial diagnosis of adult acute myeloid leukemia. Eur J Haematol 91: 411-422, 2013.

91. Aref S, El-Ghonemy MS, Abouzeid TE, El-Sabbagh AM and El-Baiomy MA: Telomerase reverse transcriptase (TERT) A1062T mutation as a prognostic factor in Egyptian patients with acute myeloid leukemia (AML). Med Oncol 31: 158, 2014

92. Akram Z, Ahmed P, Kajigaya S, Satti TM, Satti HS, Chaudhary QUN, Gutierrez-Rodrigues F, Ibanez PF, Feng X, Mahmood SK, et al: Epidemiological, clinical and genetic characterization of aplastic anemia patients in Pakistan. Ann Hematol 98: 301-312, 2019.

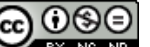

This work is licensed under a Creative Common Attribution-NonCommercial-NoDerivatives 4.0 International (CC BY-NC-ND 4.0) License. 